\title{
INTEGRATED CARE Barriers and facilitators to integrating primary and specialist healthcare in the United Kingdom: a narrative literature review
}

\author{
Authors: Olga Kozlowska, ${ }^{\mathrm{A}}$ Alistair Lumb, ${ }^{\mathrm{B}}$ Garry D $\operatorname{Tan}^{\mathrm{C}}$ and Rustam Rea ${ }^{\mathrm{D}}$
}

\begin{abstract}
Many national policies propose integration between primary and specialist care to improve the care of people with long-term conditions. There is an increasing need to understand how to practically implement such service redesign. This paper reviews the literature on the barriers to, and facilitators of, integrating primary and specialist healthcare for people with long-term conditions in the UK, with the aim of informing the development and implementation of similar initiatives in integration. MEDLINE and CINAHL databases were searched and 14 articles discussing factors hindering or enabling integration were identified. The factors were extracted and synthesised and key lessons were tabulated. Successful integration of care requires synchronised changes on different levels, a well-resourced team, a welldefined and evidence-based service, agreed and articulated new roles and responsibilities, and a willingness among healthcare professionals to co-work and co-learn. Barriers to successful implementation of integrated care include a lack of commitment across organisations, limited resources, poorly functioning information technology (IT), poor coordination of finances and care pathways, conflicting objectives, and conflict within teams. The examples of integrated working provide insights into problems and solutions around interorganisational and interprofessional working that will guide those planning integration in the future.
\end{abstract}

KEYWORDS: Integration, primary care, specialty care, healthcare, barriers, facilitators, change

\section{Introduction}

A need to integrate care for people with long-term conditions

The current structure of the health service - with primary care professionals managing access to specialist care - should in principle

Authors: ${ }^{\text {A } L o r d ~ L e o n a r d ~ a n d ~ L a d y ~ E s t e l l e ~ W o l f s o n ~ f u t u r e ~ h o s p i t a l ~}$ project fellow, Royal College of Physicians, London, UK; ${ }^{B}$ consultant in diabetes, Oxford University Hospitals NHS Foundation Trust, Oxford, UK and NIHR Oxford Biomedical Research Centre, Oxford, UK; C ${ }^{C}$ consultant in diabetes, Oxford University Hospitals NHS Foundation Trust, Oxford, UK and NIHR Oxford Biomedical Research Centre, Oxford, UK; ${ }^{D}$ consultant in diabetes, Oxford University Hospitals NHS Foundation Trust, Oxford, UK be able to offer whole-person care which allows early identification of disease, timely and appropriate interventions, and prompt management of complications. However, in reality, the current structure is fragmented with care providers working across different organisations, which are driven by different priorities, metrics, outcomes and budgets. In the context of what can be contradictory care arrangements, delivering person-centred care that is clinically effective, safe, timely, efficient and equitable is challenging.

In response, there has been a policy drive to redesign how care for people with long-term conditions is delivered. A view emphasising the role of integrated care was expressed by NHS England in Integrated care and support: our shared commitment ${ }^{1}$ and the Five Year Forward View. ${ }^{2}$ Furthermore, organisations representing healthcare professionals have also proposed ways to dissolve traditional boundaries between general practice, community services, hospitals and social care; such bodies include the British Medical Association (BMA), ${ }^{3,4}$ the Royal College of Physicians (RCP), ${ }^{5,6}$ the Royal College of General Practitioners (RCGP), ${ }^{7-10}$ and the Royal College of Nursing. ${ }^{11} \mathrm{~A}$ joint RCP and RCGP $^{12}$ statement promoted service design models which were focused on establishing joint management and delivery structures to provide coordinated care and to develop interprofessional leadership and teams spanning services and settings which are driven by shared outcomes.

These policy documents and professional bodies propose that service development and care delivery should be driven by person-centred coordinated care. They propose novel ways of closer working between different parts of the health service, and flexibility while developing new models of care (no 'one size fits all' approach). National guidelines and regulatory, financial and incentive schemes provide a starting point for organisations to develop services according to their local specifications. Without explicit and detailed programmes ready to be implemented, many organisations across the country have been testing new and innovative solutions; about 50 NHS vanguards, 25 pioneers and 15 primary care home rapid test sites have been supported by NHS England, while eight Future Hospital development sites have been supported by the RCP.

Options of integration between primary and specialty care

Integrated care is defined as "an organising principle for care delivery that aims to improve patient care and experience 
through improved coordination' with integration being 'a combined set of methods, processes and models that bring it about.'13 There is a combination of ways by which integrated care may be organised, ranging from simple linkage, through coordination, to full integration. The most complete form of integration has been recommended for the care of people with severe, complex and long-term needs, ${ }^{14}$ but such full integration does not necessarily mean organisations merging. Indeed, improving the way different parts of the health service work together around the needs of patients has been voiced as being more effective in terms of improving patient outcomes than a structural change. ${ }^{4,15}$

The ways in which different parts of the health service can seek to achieve more integrated care with partnerships and networks between organisations (virtual integration) without structural change are numerous and include:

$>$ joined-up or integrated care pathways

$>$ chains of care (the needs of patients with particular conditions are met through care pathways that link different parts of the health system; networks of providers who work together through contractual agreements with commissioners; chains of care take responsibility for the budget and as such commissioners' agreements with providers specify volume, cost, quality and method of delivery)

$>$ managed clinical networks (networks of providers but without budgetary responsibility)

$>$ care planning (co-production of a personalised care plan by a care coordinator and patient with the coordinator overseeing the plan and brokering care over multiple providers)

> case management (nurse led and community-based planning and coordinated care)

$>$ disease management (similar to case management but with a focus on populations; may include multidisciplinary discussion of care, patient and provider education)

$>$ co-location (professionals from different organisations being located in, and potentially working together from, the same place to offer a common service eg community-based or specialist outreach clinics, location of a specialist health or social worker in a primary care setting)

> contractual agreements between organisations (similar but more formal approach than that of shared guidelines or protocols; it is to guarantee adherence to joined-up activities)

$>$ multidisciplinary teams (regular communication or meetings between professionals working in different organisations, joint discussion of cases, joint decision making and multidisciplinary assessments)

$>$ shared guidelines and/or protocols

$>$ virtual teams (typically involves staff from different organisations who work together informally through networks and alliances to present a unified service; the management and the structure of each organisation participating in the virtual team remain separate)

$>$ virtual wards (combine the case management and multidisciplinary team approaches; multidisciplinary assessment of patients' care needs take place for those who have been selected via risk stratification techniques for 'admission'; their care remains home based and is coordinated by a case manager and regularly reviewed by the team with the GP maintaining overall responsibility for the patient). ${ }^{4}$
The simplified models of care involving organisational changes have been suggested with two models focusing on a range of arrangements for community providers:

> multispecialty community providers (MCP) (extended group practices forming federations, networks or single organisations offering a wide range of care using a broad range of professionals; primary care employing consultants or taking them on as partners, senior nurses, consultant physicians, geriatricians, paediatricians and psychiatrists working alongside community nurses, therapists, pharmacists, psychologists, social works and other staff with an aim of shifting the majority of outpatient consultations and ambulatory care out of hospital settings)

> primary and acute care systems (PACS) (single organisations providing list-based GP, hospital, community and mental health services). ${ }^{2}$

\section{Barriers to and facilitators of integrating care}

In their joint statement, the RCP and the RCGP recognised the need to identify local barriers to integrated working in order to inform and influence national policy. ${ }^{12}$ In this paper, we focus on barriers to and enablers of integration between primary and specialist care with consideration of the wider context of changes in the healthcare in the UK. The objective of this review is to inform the development and implementation of new integration programmes.

\section{Method}

\section{Search strategy}

CINAHL and MEDLINE databases were searched for journal articles discussing barriers to integration of primary and specialist healthcare for people with long-term conditions. Search strategy included the following terms: ((((("primary care" OR "general practice") AND ("secondary care" OR "specialist care")) AND (integrat* OR collaborat*)) AND (barrier* OR obstacle* OR challenge* OR facilitat ${ }^{*}$ OR enable* OR implement* OR adopt*)) AND (England OR Wales OR Ireland OR Scotland OR UK OR Britain OR United Kingdom)).ti,ab. The search was limited to articles published in English since 2000 and also to publications related to healthcare in the UK. The search was broad and not limited to any particular health condition to enable cross-disciplinary learning.

\section{Results}

The search of the academic databases identified 47 abstracts with 14 full-text articles included in the analysis. Thirty-three abstracts were excluded based on the pre-specified exclusion criteria: not primary/secondary care interface, not a long-term or chronic condition, or no description of facilitators or barriers to integration of care. Articles discussing issues related to problems in delivery of healthcare across primary and specialist services and suggesting implementation of integrated care were also excluded as they did not directly address the barriers or facilitators related to organisational integration.

Of the 14 articles included into the final review there was one systematic review, ${ }^{16} 12$ original research studies, and one discussion article. The publications covered six clinical areas: mental health, ${ }^{16-18}$ diabetes, ${ }^{19-21}$ chronic obstructive pulmonary 
disease, ${ }^{22,23}$ musculoskeletal condition, ${ }^{24}$ cardiovascular disorder, ${ }^{25}$ and glaucoma. ${ }^{26}$ The particular issues in healthcare management across long-term conditions included problems of working together between primary and secondary care related to poor relationships and different perceptions of priorities or standards, ${ }^{27,28}$ and the role of integrated health records in integration of care. ${ }^{29}$

Table 1 describes the studies while Table 2 summarises the barriers and facilitators identified in them.

The papers included ranged from those solely focused on barriers to, and facilitators of, integration to those giving some insight into barriers and facilitators while describing various aspects of clinical practice, clinical education or health service functioning. Assessment of the papers was challenging because of the variety of service specifications, the multiple methodologies used to evaluate integration and the level of detail included. These made drawing definite conclusions difficult.

Table 1 provides a description of the reviewed papers with a focus on determining the type of evidence considered, the study design if the paper reported on an original piece of research, the elements of integration, the outcome measures of service transformation, and any support provided to implement the new service.

\section{Barriers to and facilitators of integration of care}

The identified barriers that halted or hindered integration included:

$>$ lack of commitment to integration by the organisations involved

$>$ conflicting organisational interests

$>$ insufficient resources to develop the integrated service

$>$ inadequate mechanisms of payments between the organisations

> poor exchange of information on patient health records between healthcare professionals

$>$ poor coordination of care across the integrated service

$>$ insufficient focus on patients' needs and wishes when planning and delivering care

$>$ moving care to primary care without upskilling the workforce

$>$ tensions between healthcare professionals because of uncertainties over their new roles and responsibilities

$>$ misunderstandings over priorities in care

$>$ resistance to change.

Among the factors that supported the development and implementation of integration were:

> well-defined and evidence-based service

$>$ general practices working together

$>$ well-resourced team equipped with additional finance, time and team members

$>$ shared goals and values across organisations

> improved electronic communication

$>$ fostering commitment and enthusiasm for joint working

$>$ monitoring care quality and performance.

Table 2 summarises both the barriers to, and enablers of, integration.

\section{Discussion}

We discuss the findings from the reviewed papers in the context of the literature on integration of health and social care in the
UK. The recommendations derived from the merged literatures include.

> Appraise the options before pursuing integration.

$>$ Synchronise change at all levels of the healthcare system.

$>$ Address the unexpected consequences of change.

$>$ Engage stakeholders in developing intervention.

$>$ Train the workforce for collaborative working.

> Facilitate sharing of information, resources and patients.

This discussion focuses on the six areas above. The first three were not described in this literature review, but were identified from the wider integration literature in health and social care. The first two areas - an options appraisal and addressing change at all levels of the healthcare system - are covered only by one paper selected for this review. ${ }^{24}$ The third area of this discussion highlights the need to consider unexpected consequences of interventions; a factor rarely considered.

The last three areas arise from this literature review and cover the importance of developing the intervention collaboratively, training the workforce to work in a different way and ensuring that the infrastructure is in place to support the integrated model. If overlooked, any of these six areas may undermine the intervention; if addressed, they enable the intervention of integration to thrive. Finally, the discussion summarises the findings from the review and presents recommendations and a checklist for integrating care.

\section{Appraise the options before pursuing integration}

With policies and healthcare professional bodies promoting integration of care, there is a risk of perceiving integration as the best solution to current problems in the health services in England without fully considering why and when integration may / may not bring improvement. The Health and Social Care Act 2012 promotes provision of care in an integrated way when it is considered that it would improve the quality and outcomes of those services and reduce inequalities between persons with respect to their ability to access those services. ${ }^{30}$

The World Health Organization, ${ }^{31}$ summarising learning from the Cochrane Review, ${ }^{32}$ suggested that too often integration is seen as a quick and safe solution leading to no, or inadequate, change. There are a variety of reasons for such failure of integrated care.

> Integration is not a simple cure for a health service that doesn't work. Before implementing integration, an underlying problem should be identified and addressed with integration only if appropriate.

> Integrating everything into one package may not be necessary or beneficial. There are many variations possible on the continuum of integration.

> Integration is not a cure for inadequate resources. Integrating two separate programmes may bring savings but integrating new activities into an existing system requires adequate funding.

> There are more examples of policies in favour of integrated services than examples of actual implementation. New policies, translated into working practices, should be reflected in the documents and procedures.

> Quality of care can be adversely affected by integration. Hence it must be regularly monitored as with any change in health service. 


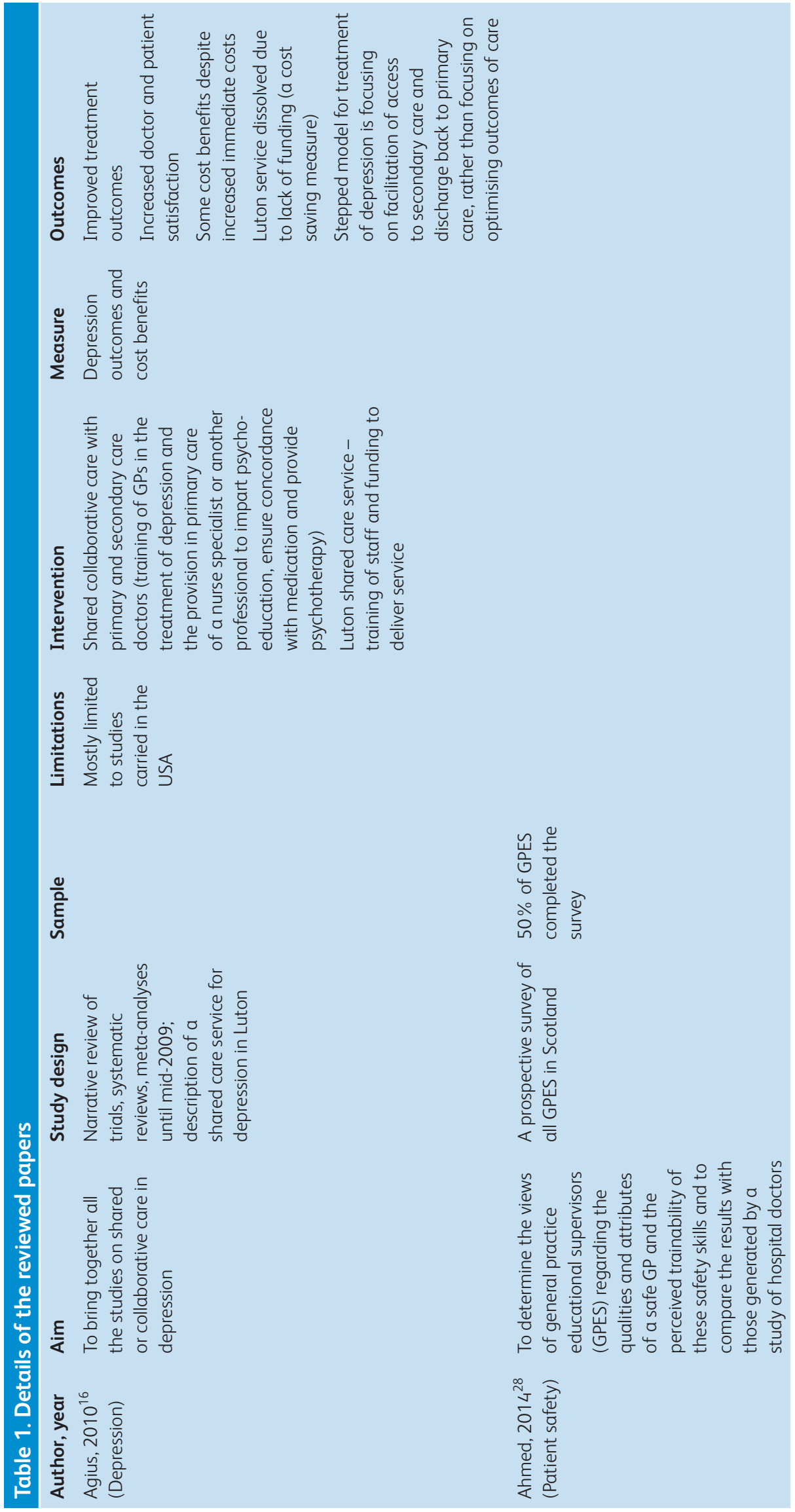




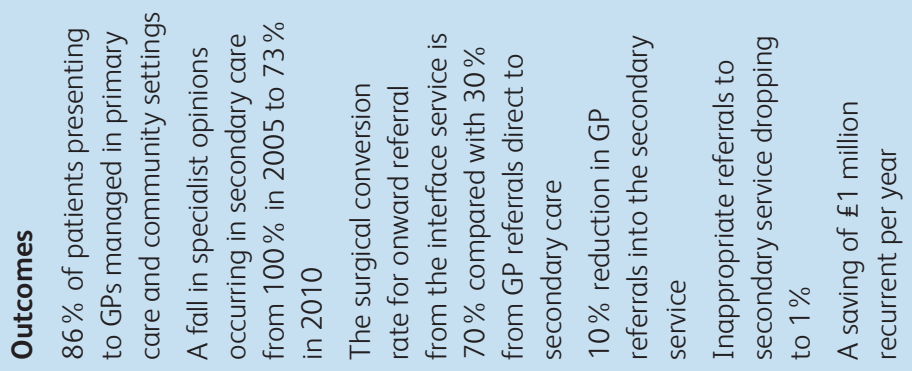

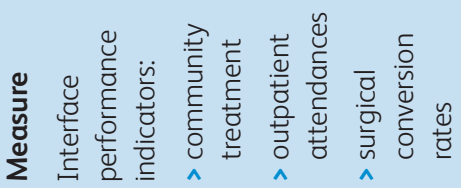

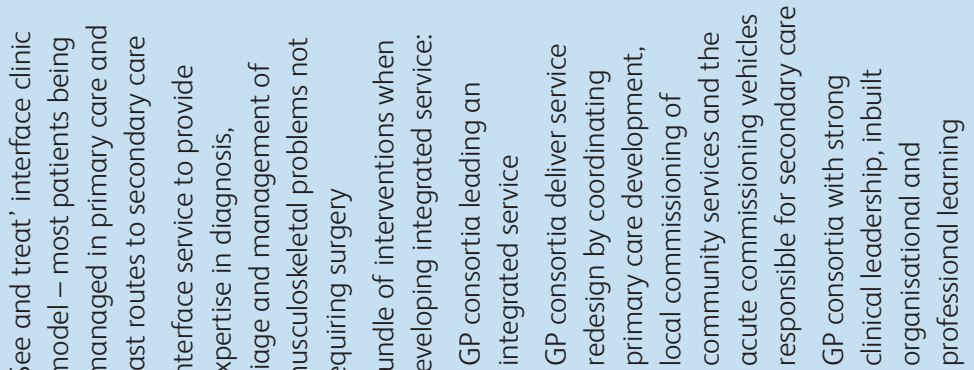
总

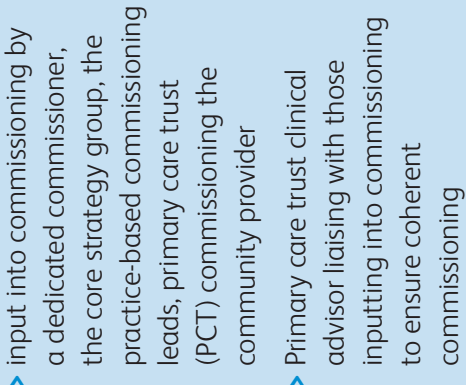

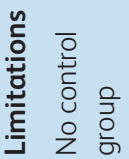

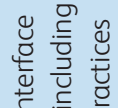

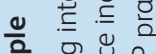

它

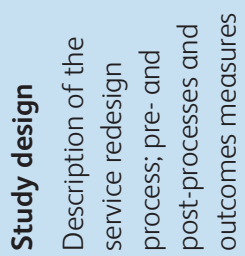

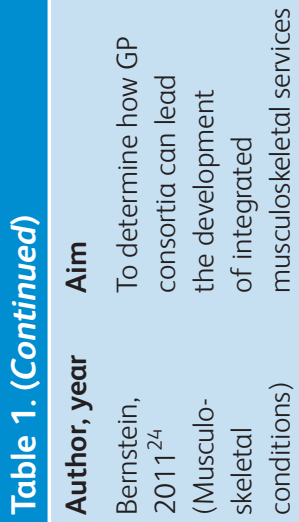




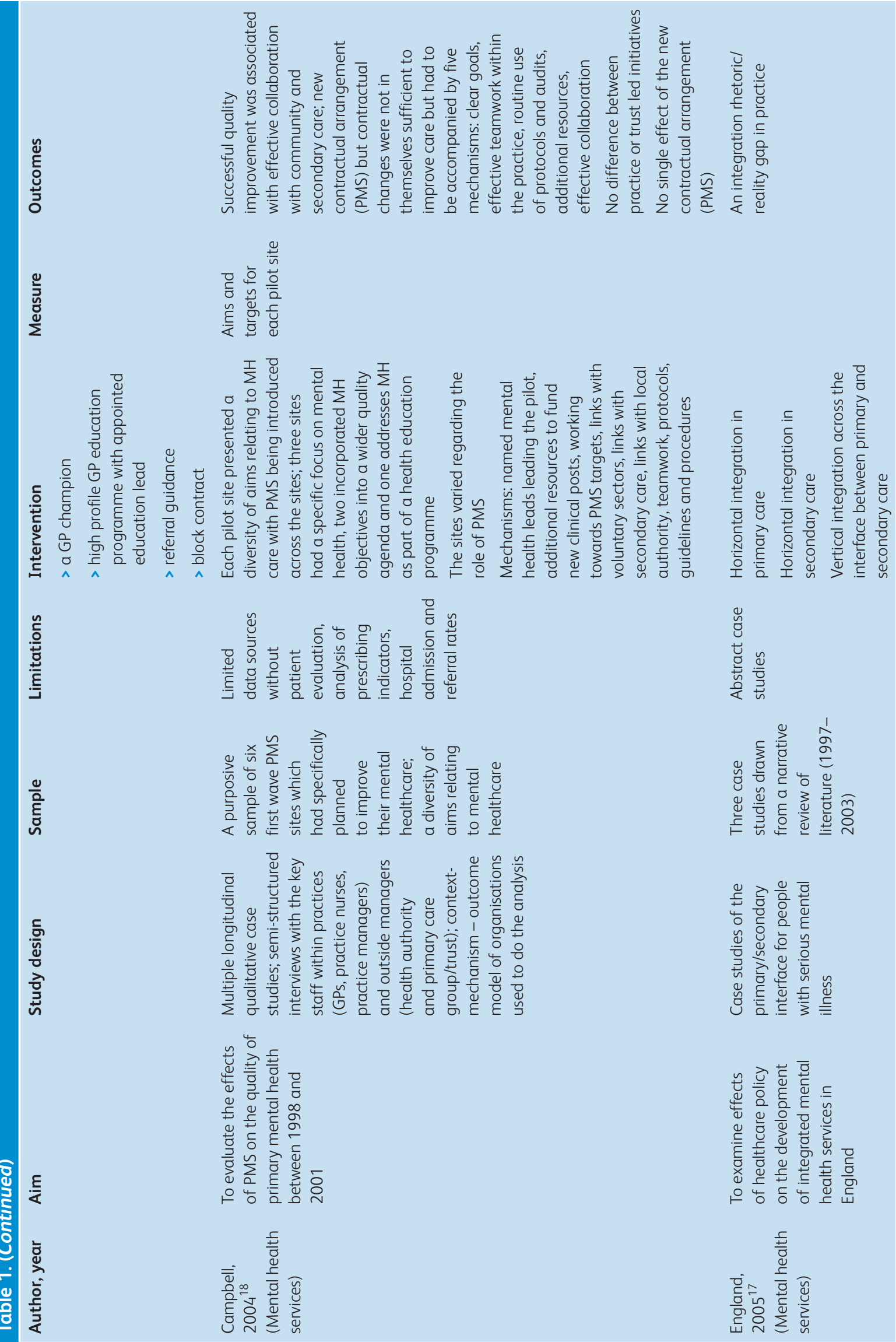



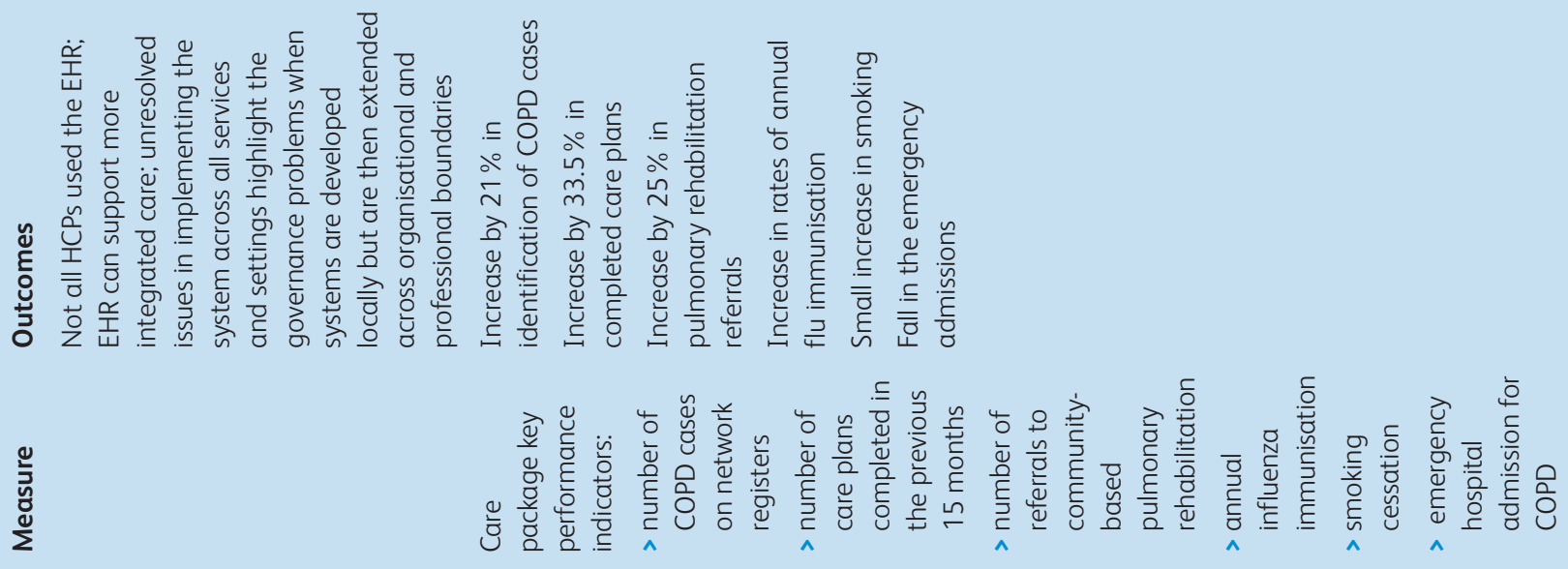

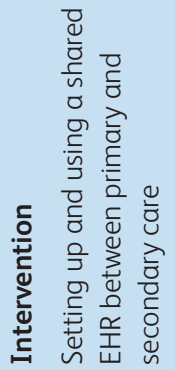

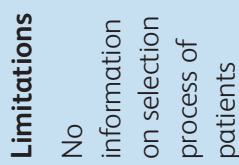

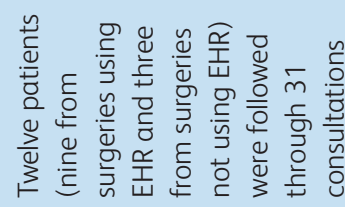
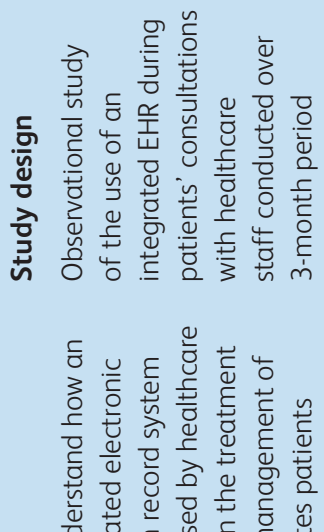

害 $\frac{E}{4}$

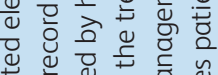
든 凉

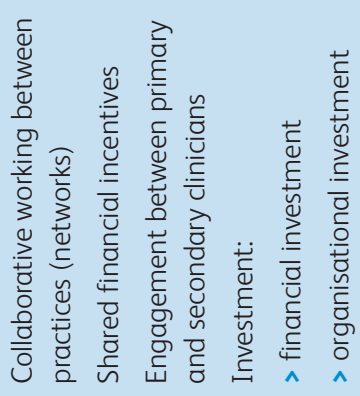

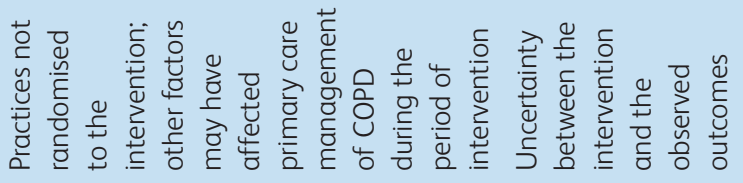
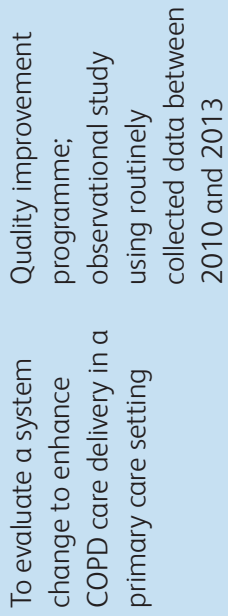

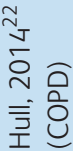




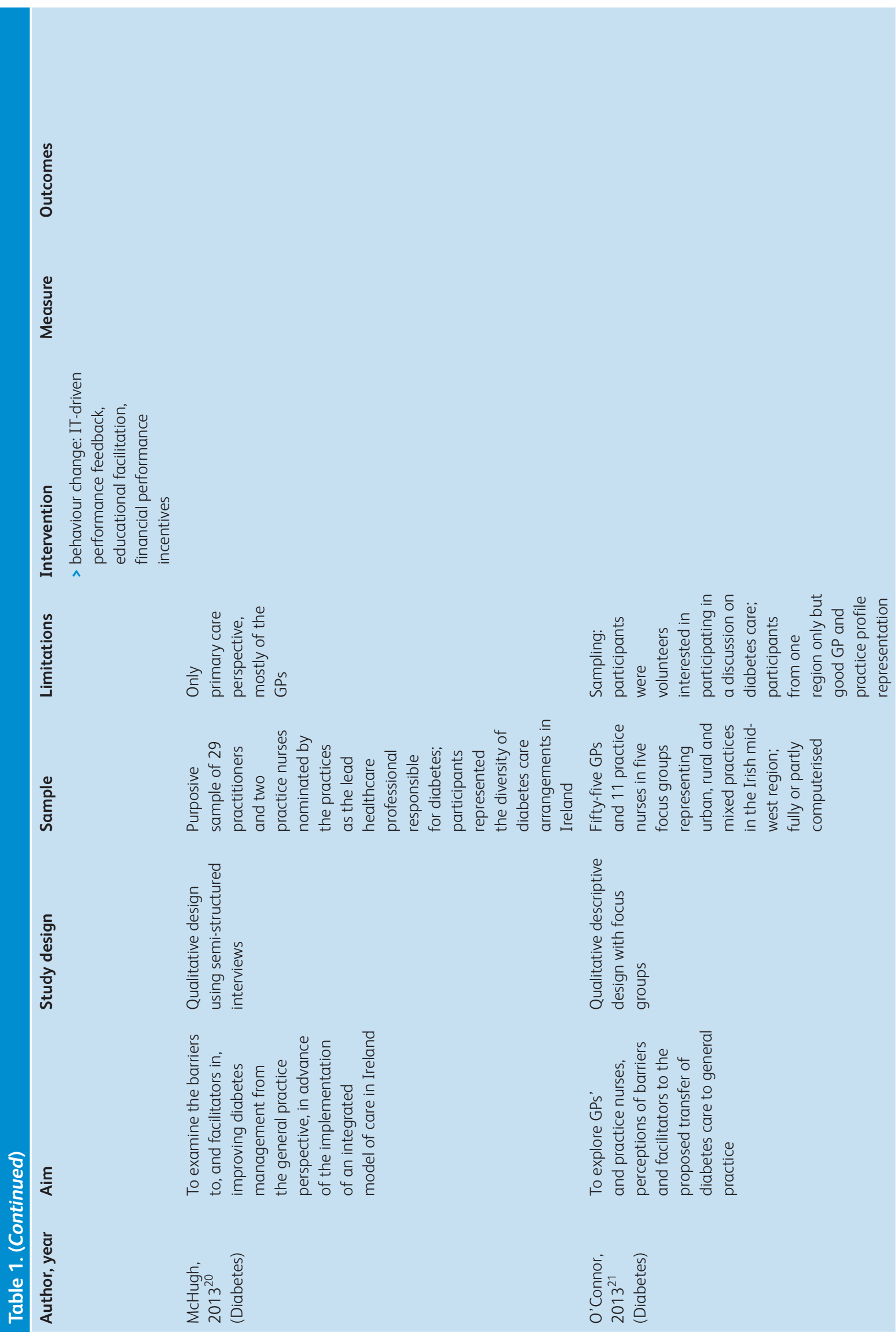




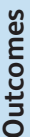 \\ $\frac{\varrho}{\grave{3}}$}

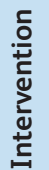

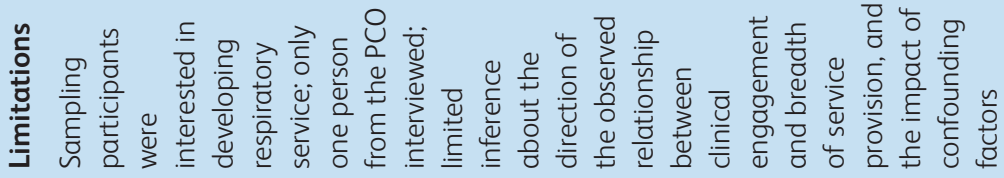

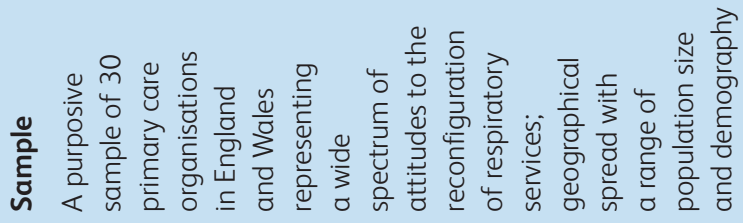

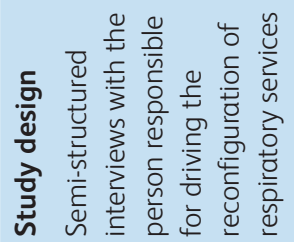
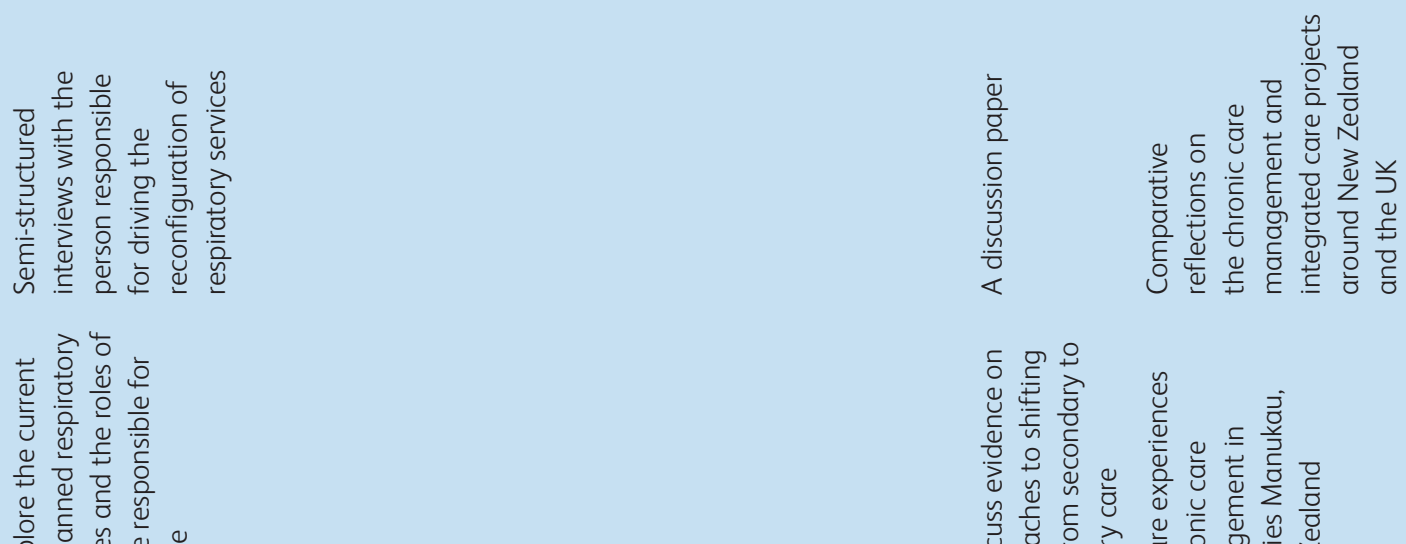

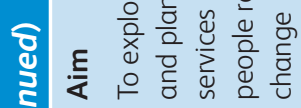

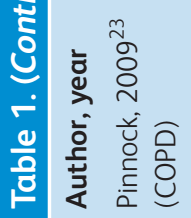
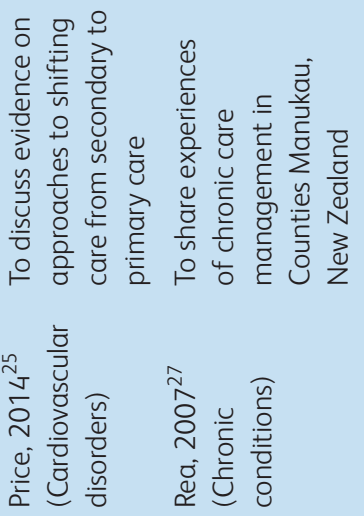


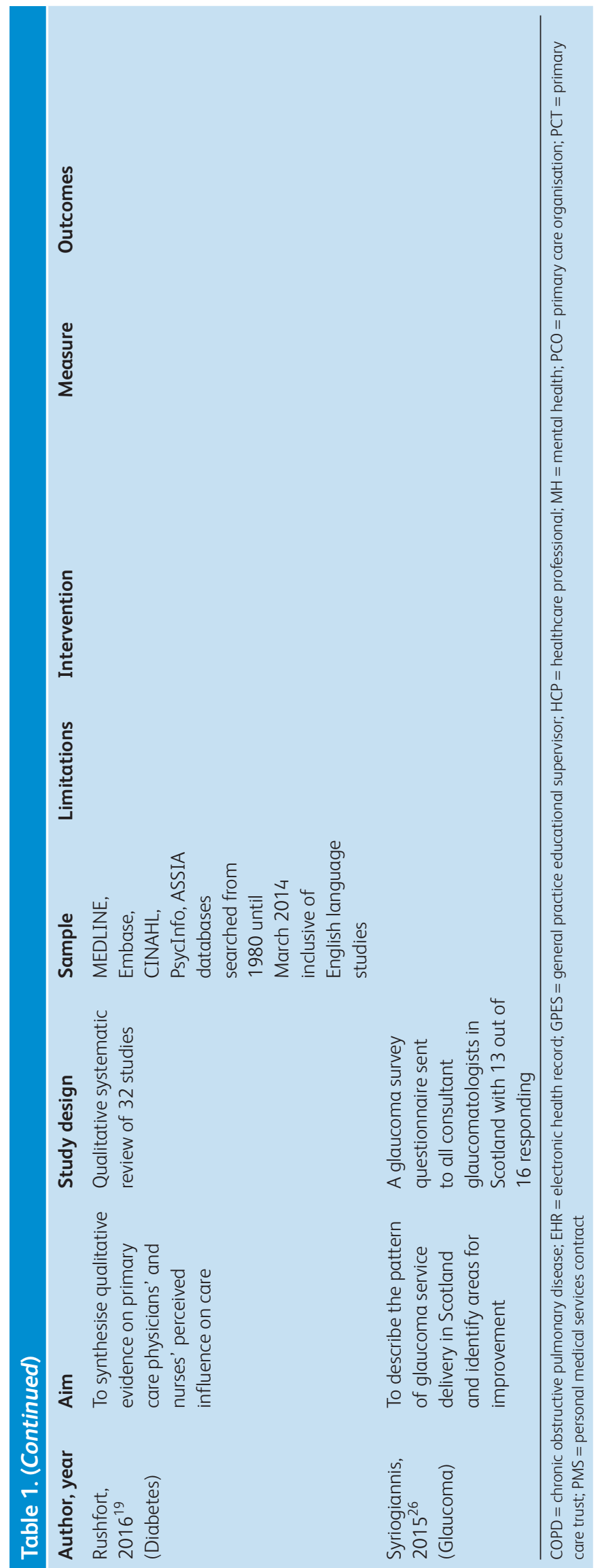


Table 2. Barriers to and facilitators of integration of care

Barriers to integration

Organisations not committed to change

\section{Organisations priortising conflicting interests}

Limited resources

Poor coordination of finance processes

Poor exchange of information between professionals

Poor coordination of care

Non-person-centered care

Lack of skills in primary care

Tensions in interprofessional teams

\section{Enablers of integration}

Strong model of care

integration
A lack of understanding of the culture of primary care

Differences in perception of core safety attributes

Defensive attitudes

Lack of interest in cooperation, entrenched attitudes and antagonism

Uncertainty over roles and responsibilities

Integration aborted due to altered service strategy

Uncertainties about health service reorganisations

Keeping the split of management and commissioning with key issue being access the Space constraints at the surgeries preventing inclinics

Financial constraints

Increase in workload and shortage of staff

Lack of targeted remuneration and financial incentives

Inadequate mechanisms for enabling the shift of finance from hospitals to community care

Difficulty to access information from secondary care

Single patient record not being used due to technical problems, compatibility problems and governance problems

Professionals individually negotiating rules for accessing patient records

Difficult to access specialist advice (GPs not knowing the consultants well enough)

coordination and communication across the interface (eg about nonrecalled by specialty service)

No consistent arrangements for discharge from primary to secondary care or shared care services in primary care

Lack of mechanisms to look into patients' needs and wishes

Paucity of formal training in primary care

Intervention based on existing guidelines

Clearly defined structure, defined actions, roles and responsibilities

Clear and fast routes to secondary care

Named lead (a GP)
Source

Campbell, $2004^{18}$

Campbell, $2004^{18}$

Agius, $2010^{16}$

Campbell, $2004^{18}$

Pinnock, $2009^{23}$

Hull, 2014; ${ }^{22}$

O'Connor, $2013^{21}$

McHugh, 2013; 20

O'Connor, $2013^{21}$

Campbell, $2004^{18}$

Campbell, $2004^{18}$

Featherstone, $2012^{29}$

Featherstone, $2012^{29}$

England, 2005 ${ }^{17}$

England, 2005; ${ }^{17}$

McHugh, $2013^{20}$

Syriogiannis, $2015^{26}$

Hull, $2014^{22}$

England, 2005; ${ }^{17}$

Pinnock, $2009^{23}$

England, $2005^{17}$

Ahmed, $2014^{28}$

England, 2005; ${ }^{17}$

Rea, $2007^{27}$

Pinnock, $2009^{23}$

England, 2005; ${ }^{17}$

Rushfort, $2016^{19}$

Price, $2014^{25}$

Price, 2014;, ${ }^{25}$

Campbell, $2004^{18}$

Bernstein, $2011^{24}$

Campbell, $2004^{18}$ 
Table 2. (Continued)

\section{Barriers to integration}

A GP champion

Clinical leadership

Interpractice working

Bringing clinicians together

Additional resources

\section{Fostering and maintaining commitment and enthusiasm for joint working}

Shared goals and values

Respect for the autonomy of the different groups involved

The surrender of professional territory where necessary

Enabling healthcare professionals to learn about each other's settings and strengths

Investment in an IT backbone to support the development of real-time information on clinical performance

Analysis of key performance indicators with clinical leads working with practice teams to support delivery

\section{Source}

Bernstein, $2011^{24}$

Campbell, 2004; ${ }^{18}$

Bernstein, 201124

Bernstein, $2011^{24}$

Hull, $2014^{22}$

Campbell, $2004^{18}$

Hull, $2014^{22}$

Hull, $2014^{22}$

Price, $2014^{25}$

Price, $2014^{25}$

McHugh, $2013^{20}$

Bernstein, $2011^{24}$

Campbell, $2004^{18}$

Campbell, $2004^{18}$

England, 2005; ${ }^{17}$

Hull, 2014; 22

Bernstein, 201124

England, $2005^{17}$

England, $2005^{17}$

England, $2005^{17}$

England, $2005^{17}$

Hull, $2014^{22}$

Hull, 2014; 22

Price, $2014^{25}$

$\mathrm{GP}=$ general practitioner; $\mathrm{MDT}=$ multidisciplinary team

Synchronise change at all levels of the healthcare system

Integration may fail when the need for change is initiated at one healthcare level without clear facilitation of that change at other organisational levels. ${ }^{33}$ Whether a top-down or bottom-up approach is taken, both carry risks to integration.

For example, in the case of a top-down approach, difficulties with integration may stem from a high-level centralised push for integration without adequate buy-in from all stakeholders, who may not be ready for change and are concerned about rushed implementation. Integration of care initiatives across the UK have usually been driven by centralised initiatives between different parts of the healthcare system with mixed results. This strategy does not always allow organisations and professionals the time needed to rationalise the change and prepare for it, leading to the immature adoption of ideas, which in turn results in a gap between integration rhetoric and reality. ${ }^{17}$ Policy can drive organisational restructure and reform but does not necessarily provide the essential ingredients for professional collaboration. ${ }^{17}$

In the case of a bottom-up approach, integration may fail when initiatives are not aligned with the priorities pursued higher up the organisational hierarchy. Local plans may be hindered by policy barriers ${ }^{34}$ and structural changes ${ }^{35}$ proscribed centrally. Unrelated organisational changes may also impact on the changes happening at the team or individual level. ${ }^{36}$ While primary and secondary care doctors may want to work together in a single team this level of integration may be hindered by separate management structures. ${ }^{16}$ As a result, healthcare professionals continue to focus on referring patients from one service to another and other organisational priorities, instead of optimising outcomes of care. The suggested way forward would be for the service to be re-structured to enable collaborative working towards shared goals. Relying on the intrinsic motivation of the healthcare professionals is not enough to drive and sustain change if the 
Table 3. Checklist

In your integrated care project, have you considered...

Initial considerations Have the reasons for underperforming in the existing arrangements been identified? Is there confidence that integration will help to address these issues?

If there is negative history of working between organisations involved, has it been recognised and discussed between the stakeholders?

Has it been considered if the existing collaboration mechanisms could be built on?

Model of care

Has the new service been designed based on evidence and guidelines?

Is the vision (structure and aims) of the new service clearly defined?

Is it clear who will lead and take responsibility for the new service?

Has the scope of the new service been agreed on?

Has the split between management and commissioning been addressed? (Is the focus on optimising care rather than gate keeping?)

Have the shared outcomes been agreed on?

Has the payment and incentive system been aligned with the shared outcomes?

Has a risk of patients using an alternative provider been considered?

Has it been considered if resources and responsibilities could be shared between the general practices?

Patient-centred care

Are there mechanisms to look into patients' needs and care preferences?

Organisation level

Has it been considered what national/local transformation programmes could be used to pursue integration?

Are the organisations involved committed to integration?

Is the organisational environment stable?

Are the interests of organisations involved in integration non-clashing?

Is there executive buy-in?

Project delivery

Have the resources to manage the project been allocated?

Is there a project management process in place?

Are the resources to achieve the project's aim sufficient?

Are managers on the integrated project facilitating and encouraging clinical engagement?

Are all organisations and stakeholders represented on the project team?

Is the vision of the integrated care service shared in the team?

Is the vision reflecting the local needs? Is there buy-in from all stakeholders?

Are the team members committed to change? Are they ready to commit their time?

Has the timescale been agreed on?

Clinical leadership

Is there a strong clinical leadership?

Is there a strong primary care leadership?

Are the clinical leads taking roles of champions and mentors?

Working together

Has working together been enabled, eg onsite clinics, MDT meetings?

Has knowledge sharing been enabled, eg formal training, MDT meetings?

Is the access to specialist advice easy and timely?

Is there confidence across primary care to access specialist advice?

Do healthcare professionals in the integrated service feel respected by each other?

Are the management styles and extent of delegation of authority consistent?

Coordination of care Are shared guidelines/protocols in place?

Is the route to specialist care clear and fast?

Are there consistent arrangements for discharge or shared care services in primary care? 
Table 3. Checklist

In your integrated care project, have you considered...

Is there a mechanism in place to indicate where the patient is in the integrated service, for how long and what are the next steps?

Has exchange of information on patient health records been enabled?

Is the care plan shared?

Operating procedures Are unified performance frameworks in place?

Is transfer of funds between organisations enabled?

Workforce planning Does primary care workforce have skills to deliver care expected in the new service?

Are new roles and responsibilities clear?

Are the healthcare professionals trained to use new IT solutions?

Are the healthcare professionals open to the new ways of working?

Has it been considered if there is a need for new skills in the system?

Has it been assured that the new arrangements will not deskill primary care?

Monitoring processes Have the care quality, processes and performance monitoring mechanism been put in place? and outcomes Is there a system in place to recognise and address unexpected consequences of change?

IT = information technology; MDT = multidisciplinary team

change is not actively supported at other levels of the system; for example, shifting diabetes care for people with uncomplicated type 2 diabetes from secondary care to primary care and sharing the management of patients with complicated type 2 diabetes between primary and secondary care without the targeted remuneration for diabetes management (decided at the health system level) and providing adequate resources in primary care (decided at the organisational level). ${ }^{20}$

\section{Address the unexpected consequences of change}

Successful integration can have unexpected negative consequences. It is common to assume that integration and its individual components will have a positive effect; however, negative (sometimes paradoxical) consequences have been identified. ${ }^{17}$ For example, the introduction of a new service aimed at complex patients in an underserved population may lead to large numbers of referrals of people with non-complex problems limiting the service's ability to adequately treat the patients for whom it was set up. Shifting outpatient clinics, enabling specialists to operate clinics within primary care surgeries, can lead to a deskilling of GPs. Attaching trained workers to primary care teams, eg community nurses to support people in primary care settings, can lead to confusion over roles and responsibilities with resulting inefficiency and both duplication and gaps in the service.

\section{Engage stakeholders in developing intervention}

A key element of successful integration is co-production of the new model of care with all stakeholders who are involved in, and affected by, care. It may be especially difficult when the scope of intervention is wide and targets multiple populations ${ }^{37}$ and also where there is a history of negative working between organisations involved. ${ }^{38}$

However, it may be unclear who is best suited to represent a particular group of interest or organisation. There are at least two issues to consider: whether it is clinicians or managers who should take this role; and who has the knowledge and skills required for service transformation. One study identified engagement of clinicians from both primary and secondary care in planning, implementation and governance of the programme as a key factor in the success of the integrated services by allowing diverse perspectives to influence the service development and aligning objectives. ${ }^{23}$ In contrast, a lack of clinical engagement has been identified as a threat to integration. The main causes for concern are a lack of interest in the new ways of working, the absence of buy-in from GPs and insufficient clinical leadership. ${ }^{23,35}$ These risks have been mitigated by managers encouraging and facilitating clinical engagement (eg nominating leads), and clinical leads assuming the role of champions and mentors. ${ }^{23}$ Practice-based commissioning was perceived as a potential mechanism of increasing clinical involvement. ${ }^{23}$

Even when stakeholder engagement happens, there can still be obstacles to good communication and relationships including personal, professional and organisational conflicts of interest and poor perception of other stakeholders. ${ }^{39,40}$ A systematic review of governance models for integrated primary/secondary care recommended pursuing a number of strategies to overcome these challenges: a shared vision focused on patient safety and quality care, a commitment to partnership, strong leadership and the implementation of necessary reforms for transformation at all levels of the healthcare system. ${ }^{40}$

\section{Train the workforce for collaborative working}

Problems can arise when healthcare professionals are not sufficiently trained or supported to collaborate in an integrated care setting. $^{17,23,36,41,42}$ From the perspective of doctors, members of the BMA shared their experiences of organisational mergers including mergers of acute trusts, PCT clusters, and community and acute trusts; they stated that the most important enablers to integration were related to ethos and relationships including collaborative culture, good professional relationships, and effective clinical leadership. ${ }^{43}$ 
Shifting specialist care to primary care without equipping the primary care professionals with the knowledge, skills and competences needed to deliver good quality care within an agreed service specification will result in demoralisation, reduced clinical engagement and outcomes that are not as good as expected. The barriers to provide education is paucity of formal training ${ }^{17}$ and a limited remit to provide education by specialists. ${ }^{23}$

Even where there is adequate education for healthcare professionals, the education and training is often segregated, and as a result does not encourage healthcare professionals to work together towards the same goal. Interprofessional education is seen an enabler to learn about each other's settings and strengths, and encourage a culture of collaboration and mutual respect. ${ }^{17}$ A specific example of the latter is education and training in health informatics for integrated care. This is particularly important given that health informatics is seen as one of the pillars of integrated care. ${ }^{44}$ Gaps have been observed in the health informatics education and training for medical, nursing and health professionals. ${ }^{45}$ It is not realistic to prepare the workforce for all informatics systems while they are educated at the preregistration level as the systems vary across the health services. However, developing some competencies at this stage may support the healthcare professionals while working in an integrated setting, including communication between multidisciplinary teams, clinical information standards, collation and management of electronic healthcare records, information governance and data protection legislation. The student training opportunities to acquire these competencies are scarce both during the medical, nursing and allied health professions preregistration education and clinical practice. $^{45}$

\section{Facilitate sharing of information, resources and patients}

Monitor (previously the UK healthcare regulator and now part of NHS Improvement) recognised a number of areas where obstacles could arise affecting willingness and/or ability to share information, resources and service users. These included:

$>$ quality of IT and communication systems

$>$ operating procedures between services (eg different performance frameworks, finance systems, planning and budgeting)

$>$ transfer of funds from one institution to another and tariff concerns

$>$ risk aversion among health professionals

$>$ service users choosing alternative providers

$>$ governance (lack of clarity over who has clinical and/or organisational responsibility)

$>$ clinical practice (differences in how to treat patients)

$>$ cultural differences (eg different management styles, extent of delegation of authority, clarity over objectives)

$>$ measurement of integrated care (lack of clear guidelines on how to measure integrated care). ${ }^{46}$

\section{Practical implications - a checklist}

Integration is a complex process which depends on a wide range of stakeholders and factors, and which is expected to occur in a health economy that is constantly changing due to new priorities, regulations and lack of resources. For those involved in integration of care, the checklist provided in Table 3, based on the review, may assist in deciding what is working well and what needs more attention. Having been alerted to key elements in integration, those leading the service transformation will need to assess, monitor, measure and evaluate the extent of integration. A number of resources are available to help achieve this (for example the NHS Improvement resources: www.improvement.nhs.uk).

\section{Conclusion}

This review of the barriers and facilitators of integrating care for long-term conditions from across the UK provides insights into the development and implementation of coordinated primary and specialty care. Three recommendations transpire from the review.

> There is a need for detailed reporting about the processes and interventions involved in the development and implementation of the integrated projects, eg Bernstein, 2011. ${ }^{24}$

> There is a need for comparative studies determining what elements of interventions are effective and in what configuration, eg Campbell, 2004. ${ }^{18}$

$>$ There is a need for a more systematic approach to the evaluation of the key enablers and barriers identified during development and implementation of integrated services. Too often the barriers/facilitators referred to as 'key' to integration are not supported by rigorous investigation.

Many authors of the reviewed papers identified 'key' factors of integration spanning the health system to the individual healthcare professional level and covering advice both relevant to managing change and improving clinical practice. The listed mechanisms acting as facilitators were usually associated with more comprehensive integration. Each mechanism may bring change but only their combination can secure full integration. The 'key' factors identified fell into the following themes.

> Strategy: overcoming interorganisational divides, ${ }^{17}$ addressing the broader strategic issues of each organisation, ${ }^{23}$ positive collaboration with health authority, hospitals, and community based secondary care. ${ }^{18}$

> Clinical engagement in the development and implementation: engagement in the planning, implementation and governance of the programme and in contributing to the educational support in the MDT meetings; ${ }^{22}$ strong clinical leadership, a core strategy group, a GP champion, all coordinating primary care development, local commissioning of community services and the acute commissioning responsible for secondary care; ${ }^{24}$ clear aims and vision shared between practice staff, community trust partners, and the health authority. ${ }^{18}$

> Interprofessional working: overcoming interprofessional differences by knowledge sharing, respect for the autonomy of the different groups involved, the surrender of professional territory where necessary: ${ }^{17}$ good teamwork; ${ }^{18}$ providing for local educational needs; ${ }^{23}$ the presence of multidisciplinary teams (integrated primary and secondary clinical teams or integrated primary and secondary care clinicians with management). ${ }^{23}$

> Coordination of care: easy access to secondary care; ${ }^{16,21}$ linked information systems. ${ }^{20}$

> Resources: remuneration for chronic disease management; ${ }^{20}$ appropriate investment in infrastructure and resources; ${ }^{18,20-22}$ time resource; ${ }^{21}$ workforce resource; ${ }^{21}$ sufficient services particularly in the community. ${ }^{20}$ 
> Clinical practice: common medication guidelines across primary and secondary care, ${ }^{16}$ shared protocols. ${ }^{18,20,21}$

$>$ Culture of learning: inbuilt organisational learning and professional learning for providers at every stage of the care pathway from general practice, interface services to secondary care, $^{24}$ real time information on clinical performance relevant to both clinicians and commissioners. ${ }^{22}$

There has been a rapid increase in the number and diversity of new models of integrated care, stimulated by the Five Year Forward View. ${ }^{2}$ The NHS England vanguard sites, including integrated PACS and MCPs, are coming to the end of their 2-year pilots after being selected in 2015. Primary and acute care systems and MCPs have demonstrated, in over 1 year, that the growth in emergency admissions and bed days from the base year has been lower than for non-new care models, but it has yet to be identified what elements have contributed to the success and what could be replicated elsewhere. ${ }^{47}$ The NHS England Integrated Care Pioneers, developing and testing new and different ways of joining up health and social care services since 2014, underwent an early evaluation to describe implementation of the programmes and barriers to and facilitators of integration. ${ }^{48}$ Although integration has been on the national agenda, the pioneers identified national issues, including the legal framework for contracting/ commissioning and information governance as persisting issues. However, the national context has been changing and those barriers are being challenged. New commissioning models include outcome-based commissioning at a population level (the Rightcare approach) and person-centred care commissioning at an individual and population level (the House of Care approach). With general practices consolidating to create more sustainable organisations, and working collaboratively through federations to deliver healthcare at scale, even up to clinical commissioning groups (CCGs) merging, new opportunities for collaboration will arise.

The current changes in commissioning with new solutions being tested around the country provide novel mechanisms of integrating care. Whether by pulling budgets between organisations providing an integrated service to increased buying power and better planning of resources or by remuneration of integrated working to motivate collaboration, the options are numerous and there is increasing flexibility in developing them locally. These solutions will, however, only change our understanding of what works for whom if there is careful analysis of the contracting process and comprehensive evaluation of the commissioning outcomes.

\section{Disclaimer}

The views expressed are those of the authors and not necessarily those of the NHS, the NIHR or the Department of Health.

\section{References}

1 National Collaboration for Integrated Care and Support. Integrated care and support: our shared commitment. London: DH, 2013.

2 NHS England. Five Year Forward View. London: NHS England, 2014.

3 British Medical Association. General practice and integration: Becoming architects of new care models in England - A paper for discussion. London: BMA, 2015.

4 British Medical Association. Integrating services without structural change. London: BMA, 2012
5 Royal College of Physicians. Putting the pieces together: Removing the barriers to excellent patient care. London: RCP, 2015.

6 Future Hospital Commission. Future hospital: Caring for medical patients. London: RCP, 2013,

7 Royal College of General Practitioners. An inquiry into patient centred care in the 21st century: Implications for general practice and primary care. London: RCGP, 2014.

8 Royal College of General Practitioners. The 2022 GP. A Vision for General Practice in the future NHS. London: RCGP, 2013.

9 Royal College of General Practitioners General practice and the integration of care: an RCGP policy report. London: RCGP, 2012.

10 Mathers N, Patel V, Thomas M. General Practice and the Integration of Care: An RCGP Policy Report. London: RCGP, 2012.

11 Royal College of Nursing. Integrated health and social care in England: the story so far. London: RCN, 2014.

12 Royal College of Physicians and Royal College of General Practitioners. Joint statement on integrated care. London: RCP and RCGP, 2014.

13 Shaw S, Rosen R, Rumbold B. What is integrated care? Nuffield Trust, 2011.

14 Leutz WN. Five Laws for Integrating Medical and Social Services: Lessons from the United States and the United Kingdom. Milbank Q 1999;77:77-110.

15 Curry N, Ham C. Clinical and service integration. The route to improved outcomes. London: The King's Fund, 2010.

16 Agius M, Murphy CL, Zaman R. Does shared care help in the treatment of depression? Psychaitr Danub 2010;22:18-22.

17 England $\mathrm{E}$, Lester $\mathrm{H}$. Integrated mental health services in England: a policy paradox. Int J Integr Care 2005;5:e24.

18 Campbell SM, Robinson J, Steiner A, Webb D, Roland MO. Improving the quality of mental health services in Personal Medical Services pilots: a longitudinal qualitative study. Qual Saf Health Care 2004:13:115-20.

19 Rushfort B, McCrorie C, Glidwell L, Midlgley E, Foy R. Barriers to effective management of type 2 diabetes in primary care. $\mathrm{Br}$ J Gen Pract 2016;66:e114-27

20 Mc Hugh S, O’Mullane M, Perry IJ, Bradley C. Barriers to, and facilitators in, introducing integrated diabetes care in Ireland: a qualita tive study of views in general practice. BMJ Open 2013;3:e003217

21 O'Connor R, Mannix M, Mullen ] et al. Structured care of diabetes in general practice: a qualitative study of the barriers and facilitators. Ir Med J 2013;106:77-80.

22 Hull S, Mathur R, Lloyd-Owen S, Round T, Robson J. Improving outcomes for people with COPD by developing networks of general practices: evaluation of a quality improvement project in east London. NPJ Prim Care Respir Med 2014;24:14082

23 Pinnock H, Huby G, Tierney A et al. Is multidisciplinary teamwork the key? A qualitative study of the development of respiratory services in the UK. J R Soc Med 2009;102:378-90.

24 Bernstein I. Integrated musculoskeletal service design by GP consortia. London J Prim Care 2011:4:16-26.

25 Price E, Baker R, Krause J, Keen C. Organisation of services for people with cardiovascular disorders in primary care: transfer to primary care or to specialist-generalist multidisciplinary teams? BMC Fam Pract 2014;15:158.

26 Syrogiannis A, Rotchford AP, Agarwal PK et al. Glaucomaservice provision in Scotland: introduction and need for Scottish Intercollegiate Guidelines Network guidelines. Clin Ophthalmol 2015;9:1835-43.

27 Rea H, Kenealy $\mathrm{T}$, Wellingham J et al. Chronic care management evolves towards integrated care in counties Manukau, New Zealand. The New Zealand Medicine 2007;120:1252.

28 Ahmed M, Sonal A, McKay J. Patient safety skills in primary care: a national survey of GP educators. BMC Family Pract 2014;15:206.

29 Featherstone I, Keen J. Do integrated record systems lead to integrated services? An observational study of a multiprofessional system in a diabetes service. Int ] Med Inform 2012;81:45-52. 
30 Health and Social Care Act 2012, c.7 www.legislation.gov.uk/ ukpga/2012/7/pdfs/ukpga_20120007_en.pdf [Accessed 2 October 2017].

31 World Health Organization. Integrated health services - what and why? WHO, 2008.

32 Briggs C], Garner P. Strategies for integrating primary health services in middle- and low-income countries at the point of delivery (Review). Wiley, 2006.

33 Ferlie EB, Shortell SM. Improving the quality of healthcare in the United Kingdom and the United States: a framework for change. Milbank Q 2001;79:281-315.

34 Ham C, Smith J. Removing policy barriers to integrated care in England. London: The Nuffield Trust, 2010.

35 NHS Future Forum. Integration. A report from the NHS Future Forum. London: DH, 2012.

36 RAND Europe, Ernst \& Young LLP. National Evaluation of the Department of Health's Integrated Care Pilots. London: DH, 2012.

37 Ling T, Brereton L, Conklin A, Newbould J, Roland M. Barriers and facilitators to integrating care: experiences from the English Integrated Care Pilots. Int J Integr Care 2012:12:E129.

38 Miller $\mathrm{R}$, Brown $\mathrm{H}$, Mangan C. Integrated care in action. A practical guide for health, social care and housing support. London: Jessica Kingsley Publishers, 2016.

39 Nail YJ, Anderton C, Fell G. Integrating specialist advice following reforms: an interview-based survey. JRSM Short Rep 2013:4:24.

40 Nicholson C, Jackson C, Marley J. A governance model for integrated primary/secondary care for the health-reforming first world results of a systematic review. BMC Health Serv Res 2013:13:528.

41 Hussain S, Dornhorst A. Integrated care - taking specialist medical care beyond hospital walls. A report to the Royal College of Physicians Future Hospital Programme. London: RCP, 2016.
42 NHS England. Integrated Care Pioneers: One Year On. London: NHS England, 2015.

43 Knight M. Doctor's perspectives of organisational mergers. British Medical Association. 2012. www.docplayer.net/22662173-Doctorsperspectives-of-organisational-mergers.html [Accessed 2 October 2017].

44 NHS Diabetes. Best practice for commissioning diabetes services. An integrated care framework. 2013.

45 Bartholomew $\mathrm{N}$. Is higher education ready for the information revolution? International Journal of Therapy and Rehabilitation. 2011:18:558-66.

46 Frontier Economics. Enablers and barriers to integrated care and implications for Monitor. A report prepared for Monitor. London: Frontier Economics, 2012.

47 Charles Tallack: Evidence emerging from evaluation of the new care models, 2017. www.kingsfund.org.uk/audio-video/charlestallack-evaluation-new-care-models [Accessed 2 October 2017].

48. Erens B, Wistow G, Mounier-Jack S et al. Early evaluation of the Integrated Care and Support Pioneers Programme. Final report. PIRU. 2015. http://piru.ac.uk/assets/files/Early_evaluation_of_IC_ Pioneers_Final_Report.pdf [Accessed 2 October 2017].

Address for correspondence: Dr Olga Kozlowska, Royal College of Physicians, London NW1 4LE, UK

Email: olga.kozlowska@icloud.com

\section{NGC Nationa \\ Guideline \\ Centre}

\section{New to systematic reviewing, or need to improve your critical appraisal skills?}

Our intensive 1-day courses will introduce you to the key principles of critical appraisal and systematic reviewing, and equip you with the basic skills to put your knowledge into practice.

$>$ Introduction to critical appraisal > Systematic reviews and meta-analysis in action

Discount available if both courses are booked together.

'Informative and practical' Dr Rajeswari Ramaraj

\section{NGC Short courses}

y 10 